\title{
Quantum gauge symmetry of reducible gauge theory
}

\author{
Manoj Kumar Dwived: $*$ \\ Department of Physics, Banaras Hindu University, \\ Varanasi-221005, India.
}

\begin{abstract}
We derive the gaugeon formalism of the Kalb-Ramond field theory, a reducible gauge theory, which discusses the quantum gauge freedom. In gaugeon formalism, theory admits quantum gauge symmetry which leaves the action form-invariant. The BRST symmetric gaugeon formalism is also studied which introduces the gaugeon ghost fields and gaugeon ghosts of ghosts fields. To replace the Yokoyama subsidiary conditions by a single Kugo-Ojima type condition the virtue of BRST symmetry is utilized. Under generalized BRST transformations, we show that the gaugeon fields appear naturally in the reducible gauge theory.
\end{abstract}

\section{INTRODUCTION}

In the standard formalism of canonical quantization of gauge theories, we can not consider the quantum gauge freedom. Since the quantum theory is defined only after fixing the gauge. However, such gauge fixing breaks the local gauge invariance. Yokoyama introduces a wider framework to quantize the gauge theories, called as gaugeon formalism, by considering the quantum gauge transformation [ 1 [ 6 ]. The main idea of gaugeon formalism is to introduce some extra (quantum) fields in Lagrangian density which are called as gaugeon fields. The resulting gaugeon Lagrangian density remains invariant under quantum gauge transformation. This formulation was originally proposed for quantum electrodynamics to settle the problem of renormalization of gauge parameter. Within gaugeon formalism, the occurrence of a shift in gauge parameter during renormalization [7] was addressed naturally by connecting theories in two different gauges within the same family by a $q$-number gauge transformation [1]. This formalism has also applied in Yang-mills theory. Since the gaugeon modes contain negative normed states that give a negative probability. So to remove this unphysical gaugeon modes, Yokoyama used a Gupta-Bleuler type subsidiary condition. But this condition is not valid if an interaction is present for gaugeon fields. With the help of BRST charge, the Yokoyama subsidiary condition can be replaced by a Kugo-Ojima type restrictions [8 11]. The gaugeon formalism have been studied in various contexts [8, 9, 12 19] but never for reducible gauge theory. This provides us an opportunity to generalize the results which is the motivation of present work.

The study the gauge theories of Abelian rank-2 antisymmetric tensor fields is important due to various reasons. Kalb and Ramond were the first who gave the idea of interaction of classical strings with Abelian rank-2 antisymmetric tensor fields [20]. Application of this interaction can be seen in Lorentz covariant description of vortex motion in an irrotational, incompressible fluid 21] and to the dual formulation of the Abelian Higgs model 22]. Importance of Abelian rank-2 antisymmetric tensor fields have been studied in supergravity multiplets [23], excited states of quantized superstring theories and anomaly cancellation in certain superstring theory 24]. Further this Abelian rank-2 antisymmetric tensor field generates effective mass for an Abelian vector gauge field through a topological coupling between these two fields 25]. Abelian rank-2 antisymmetric tensor field have been also studied for U(1) gauge theory in loop space [26]. Covariant quantization of this field was first attempted by Townsend [27] and has been studied by many authors [28, 29]. Application of this theory can also be seen in a superspace formulation where Ward-Takahashi identities are derived. [30].

On the other hand, the Becchi-Rouet-Stora-Tyutin (BRST) formulation [31, 32] is a subject of vast interests [33, 34], as it leads to unitarity and renormalizability of the gauge theories. The BSRT symmetry

*Electronic address: manojdwivedi84@gmail.com 
has found dual analogue of Hodge theory [35, 36]. The generalization of BRST transformation by making the transformation parameter finite and field dependent, known as FFBRST transformation [37, 38], has found many interests in gauge theories 38 -44]. For example, the FFBRST symmetry gets relevance in $W$-algebra of higher derivative theory [41]. The FFBRST transformation connects various solution of quantum master equation of super-conformal Chern-Simons theory [42]. The FFBRST connects various gauges in Thirring model [43]. The FFBRST is studied for maximally supersymmetric M-theories [45]. The problem of graviton propagator divergences is a also addressed [46]. The supersymmetry is also generalized by making transformation parameter finite and field dependent [47. The finite BRST-antiBRST symmetry to the case of general gauge theories is discussed [48, 49]. The generalization of parameters to the case of arbitrary Grassmann-odd field-dependent parameters is also studied [50]. In present work, we would like to study the FFBRST transformation in gaugeon formulation of reducible gauge theory.

In this paper, we study the gaugeon formalism of reducible (2-form) gauge theory. For this, we first consider Lagrangian density for Abelian rank-2 antisymmetric tensor field theory in $(3+1)$ dimensions in Landau gauge. The Lagrangian density celebrates BRST and anti-BRST transformations. Further, to study the quantum gauge transformations of the theory, we extend the configuration space of theory by including some extra (quantum) fields, known as gaugeon fields, in Lagrangian density. Within formalism, the extended Lagrangian density remains form invariant under quantum gauge transformation. However, in extended Lagrangian density the shifted fields under quantum gauge transformation satisfy same equations of motion as the original fields. But under this transformations the gauge parameter gets shifted. In this way, we can claim that the gauge parameter renormalization problem is removed for Abelian 2form gauge theory. Since gaugeon fields are unphysical, so we use Kugo-Ojima and Gupta Bleuler type of subsidiary conditions in order to remove the unphysical gauge and gaugeon modes. Further, we construct the BRST symmetric gaugeon Lagrangian density for Abelian 2-form gauge theory by introducing ghost fields corresponding to gaugeon fields. Such Lagrangian density remains invariant under both the BRST and the quantum gauge transformations. We show that in BRST symmetric gaugeon Lagrangian density both the Kugo-Ojima and the Gupta Bleuler type subsidiary conditions are converted in to a single Kugo-Ojima type condition. It is well-known that Kugo-Ojima type restriction is more acceptable than Gupta-Blueler type. We found that the BRST charge corresponding to BRST symmetry annihilates the physical states when operates on it and remains invariant under quantum gauge transformation also. Further, we generalize the BRST transformation to FFBRST transformation and show that the gaugeon fields can be generated naturally by calculating the Jacobian of path integral.

This paper is presented in following way. In sec. II, we discuss the preliminaries of Abelian rank-2 tensor field theory as a reducible gauge theory. In Sec. III, we analyses the quantum gauge freedom of theory through gaugeon formalism. Further, the improved BRST symmetric gaugeon formalism is discussed in Sec. IV. In section V, the basic FFBRST methodology is discussed. The appearance of gaugeon fields in theory through FFBRST transformations is shown in section VI. The conclusions are given in the last section.

\section{ABELIAN RANK-2 TENSOR FIELD THEORY: A REDUCIBLE GAUGE THEORY}

We start with the kinetic part of Lagrangian density for the Abelian gauge theory for rank-2 antisymmetric tensor field $B_{\mu \nu}$ is defined by

$$
\mathcal{L}_{0}=\frac{1}{12} F_{\mu \nu \rho} F^{\mu \nu \rho} .
$$

Here field strength tensor is defined as $F_{\mu \nu \rho} \equiv \partial_{\mu} B_{\nu \rho}+\partial_{\nu} B_{\rho \mu}+\partial_{\rho} B_{\mu \nu}$, which is invariant under the following gauge transformation $\delta B_{\mu \nu}=\partial_{\mu} \zeta_{\nu}-\partial_{\nu} \zeta_{\mu}$, where $\zeta_{\mu}(x)$ is a vector gauge parameter .

To quantize this theory using BRST transformation, it is necessary to introduce the following ghost and auxiliary fields: anticommuting vector fields $\rho_{\mu}$ and $\tilde{\rho}_{\mu}$, a commuting vector field $\beta_{\mu}$, anticommuting scalar fields $\chi$ and $\tilde{\chi}$, and commuting scalar fields $\sigma, \varphi$, and $\tilde{\sigma}$. The BRST transformation is then defined for $B_{\mu \nu}$ by replacing $\zeta_{\mu}$ in the gauge transformation by the ghost field $\rho_{\mu}$. 
The complete effective Lagrangian density for this theory in covariant gauge, using Faddeev-Popov formulation, is given by

$$
\mathcal{L}_{\text {eff }}^{L}=\mathcal{L}_{0}+\mathcal{L}_{g f}+\mathcal{L}_{g h},
$$

with following gauge fixing and ghost terms:

$$
\begin{aligned}
\mathcal{L}_{g f}+\mathcal{L}_{g h} & =-i \partial_{\mu} \tilde{\rho}_{\nu}\left(\partial^{\mu} \rho^{\nu}-\partial^{\nu} \rho^{\mu}\right)+\partial_{\mu} \tilde{\sigma} \partial^{\mu} \sigma+\beta_{\nu}\left(\partial_{\mu} B^{\mu \nu}+\lambda_{1} \beta^{\nu}-\partial^{\nu} \varphi\right) \\
& -i \tilde{\chi} \partial_{\mu} \rho^{\mu}-i \chi\left(\partial_{\mu} \tilde{\rho}^{\mu}-\lambda_{2} \tilde{\chi}\right),
\end{aligned}
$$

where $\lambda_{1}$ and $\lambda_{2}$ are gauge parameters. This effective action is invariant under following BRST $\left(s_{b}\right)$ and anti-BRST $\left(s_{a b}\right)$ variations:

$$
\begin{aligned}
s_{b} B_{\mu \nu} & =\left(\partial_{\mu} \rho_{\nu}-\partial_{\nu} \rho_{\mu}\right), & & \\
s_{b} \rho_{\mu} & =-i \partial_{\mu} \sigma, & & s_{b} \sigma=0, \\
s_{b} \tilde{\rho}_{\mu} & =i \beta_{\mu}, & & s_{b} \beta_{\mu}=0, \\
s_{b} \tilde{\sigma} & =\tilde{\chi}, & & s_{b} \tilde{\chi}=0, \\
s_{b} \varphi & =\chi, & & s_{b} \chi=0,
\end{aligned}
$$

and

$$
\begin{aligned}
s_{a b} B_{\mu \nu} & =\left(\partial_{\mu} \tilde{\rho}_{\nu}-\partial_{\nu} \tilde{\rho}_{\mu}\right), & & \\
s_{a b} \tilde{\rho}_{\mu} & =-i \partial_{\mu} \tilde{\sigma}, & & s_{a b} \tilde{\sigma}=0, \\
s_{a b} \rho_{\mu} & =-i \beta_{\mu}, & & s_{a b} \beta_{\mu}=0, \\
s_{a b} \sigma & =\chi, & & s_{a b} \chi=0, \\
s_{a b} \varphi & =-\tilde{\chi}, & & s_{a b} \tilde{\chi}=0 .
\end{aligned}
$$

The anti-BRST variations are similar to the BRST variations, where the role of ghost and antighost field is interchanged with some modification in coefficients.

\section{ABELIAN RANK-2 TENSOR FIELD THEORY IN GAUGEON FORMALISM}

In this section, we study the Yokoyama gaugeon formalism to analyse the quantum gauge freedom for the Abelian rank-2 tensor field theory. To analyse the gaugeon formalism for Abelian rank-2 tensor field theory, let us start with the effective Lagrangian density in $3+1$ dimension in Landau gauge

$$
\begin{aligned}
\mathcal{L}_{Y} & =\frac{1}{12} F_{\mu \nu \rho} F^{\mu \nu \rho}-i \partial_{\mu} \tilde{\rho}_{\nu}\left(\partial^{\mu} \rho^{\nu}-\partial^{\nu} \rho^{\mu}\right)+\partial_{\mu} \tilde{\sigma} \partial^{\mu} \sigma+\beta_{\nu}\left(\partial_{\mu} B^{\mu \nu}-\partial^{\nu} \varphi\right)+\epsilon\left(Y_{\nu}^{\star}+\alpha \beta_{\nu}\right)^{2} \\
& -\left(\partial_{\mu} Y_{\nu}^{\star}-\partial_{\nu} Y_{\mu}^{\star}\right) \partial^{\mu} Y^{\nu}-i \tilde{\chi} \partial_{\mu} \rho^{\mu}-i \chi\left(\partial_{\mu} \tilde{\rho}^{\mu}-\lambda_{2} \tilde{\chi}\right),
\end{aligned}
$$

where $Y_{\nu}$ and $Y_{\nu}^{\star}$ are the gaugeon fields respectively.

The Lagrangian density (6) is invariant under following BRST transformations:

$$
\begin{aligned}
\delta_{b} B_{\mu \nu} & =\left(\partial_{\mu} \rho_{\nu}-\partial_{\nu} \rho_{\mu}\right) \delta \lambda, & & \\
\delta_{b} \rho_{\mu} & =-i \partial_{\mu} \sigma \delta \lambda, & & \delta_{b} \sigma=0, \\
\delta_{b} \tilde{\rho}_{\mu} & =i \beta_{\mu} \delta \lambda, & & \delta_{b} \beta_{\mu}=0, \\
\delta_{b} \tilde{\sigma} & =\tilde{\chi} \delta \lambda, & & \delta_{b} \tilde{\chi}=0, \\
\delta_{b} \varphi & =\chi \delta \lambda, & & \delta_{b} \chi=0,
\end{aligned}
$$

and

$$
\delta_{b} Y=0, \quad \delta_{b} Y_{\star}=0 .
$$


Now, we demonstrate the following quantum gauge transformation under which the Lagrangian density (6) remains form-invariant :

$$
\begin{aligned}
& B_{\mu \nu} \longrightarrow \hat{B}_{\mu \nu}=B_{\mu \nu}+\tau\left(\partial_{\mu} Y_{\nu}-\partial_{\nu} Y_{\mu}\right), \quad \rho_{\mu} \longrightarrow \hat{\rho}_{\mu}=\rho_{\mu}, \\
& \sigma \longrightarrow \hat{\sigma}=\sigma, \quad \tilde{\rho}_{\mu} \longrightarrow \hat{\tilde{\rho}}_{\mu}=\tilde{\rho}_{\mu}, \quad \beta_{\mu} \longrightarrow \hat{\beta}_{\mu}=\beta_{\mu}, \\
& \tilde{\sigma} \longrightarrow \hat{\tilde{\sigma}}=\tilde{\sigma}, \quad \tilde{\chi} \longrightarrow \hat{\tilde{\chi}}=\tilde{\chi}, \quad \varphi \longrightarrow \hat{\varphi}=\varphi, \\
& \chi \longrightarrow \hat{\chi}=\chi, \quad Y_{\nu} \longrightarrow \hat{Y}_{\nu}=Y_{\nu}, \quad Y_{\nu}^{\star} \longrightarrow \hat{Y}_{\nu}^{\star}=Y_{\nu}^{\star}-\tau \beta_{\nu},
\end{aligned}
$$

where $\tau$ is an infinitesimal transformation parameter. The form-invariance of the Lagrangian density (6) under the quantum gauge transformation (9) reflects the following shift in parameter:

$$
\alpha \longrightarrow \hat{\alpha}=\alpha+\tau \alpha .
$$

Further, according to Yokoyama, to remove the unphysical gauge and gaugeon modes from the theory and to define physical states we impose two subsidiary conditions (the Kugo-Ojima type and Gupta-Bleuler type) which are given as [2]

$$
\begin{aligned}
\left.Q_{b} \mid \text { phys }\right\rangle & =0, \\
\left.\left(Y_{\nu}^{\star}+\alpha B_{\nu}\right)^{(+)} \mid \text {phys }\right\rangle & =0
\end{aligned}
$$

where $Q_{b}$ is the BRST charge. The expression for BRST charge using Noether's theorem is calculated as

$$
Q_{b}=\int d^{3} x\left[-2 F^{0 \nu \rho}\left(\partial_{0} \rho_{\nu}-\partial_{\nu} \rho_{0}\right)+\beta_{\nu}\left(\partial^{0} \rho^{\nu}-\partial^{\nu} \rho^{0}\right)-\partial_{\nu} \sigma\left(\partial^{0} \tilde{\rho}^{\nu}-\partial^{\nu} \tilde{\rho}^{0}\right)+\tilde{\chi} \partial^{0} \sigma-\chi B^{0}\right] .
$$

The Kugo-Ojima type subsidiary condition is subjected to remove the unphysical modes corresponding to gauge field from the total Fock space. However, the Gupta-Bleuler type condition is used to remove the unphysical gaugeon modes from the physical states. The second subsidiary condition is valid when the combination $\left(Y_{\nu}^{\star}+\alpha B\right)$ satisfies the following free equation [2]

$$
\partial_{\mu} \partial^{\mu}\left(Y_{\nu}^{\star}\right)=0,
$$

which we have derived using equations of motion. The free equation (13) guarantees the decomposition of $\left(Y_{\nu}^{\star}+\alpha B\right)$ in positive and negative frequency parts. Consequently, the subsidiary conditions (11) warrant the positivity of the semi-definite metric of our physical state-vector space

$$
\langle\text { phys }| \text { phys }\rangle \geq 0 \text {, }
$$

and hence, we have a desirable physical subspace on which our unitary physical $S$-matrix exists.

\section{BRST SYMMETRIC GAUGEON FORMALISM}

In this section we discuss the BRST symmetric gaugeon formalism for Abelian 2-form gauge theory. For this purpose we first define the Lagrangian density of such model as following:

$$
\begin{aligned}
\mathcal{L}_{B Y} & =\frac{1}{12} F_{\mu \nu \rho} F^{\mu \nu \rho}-i \partial_{\mu} \tilde{\rho}_{\nu}\left(\partial^{\mu} \rho^{\nu}-\partial^{\nu} \rho^{\mu}\right)+\partial_{\mu} \tilde{\sigma} \partial^{\mu} \sigma+\beta_{\nu}\left(\partial_{\mu} B^{\mu \nu}-\partial^{\nu} \varphi\right) \\
& +\epsilon\left(Y_{\nu}^{\star}+\alpha \beta_{\nu}\right)^{2}-\left(\partial_{\mu} Y_{\nu}^{\star}-\partial_{\nu} Y_{\mu}^{\star}\right) \partial^{\mu} Y^{\nu}-i \tilde{\chi} \partial_{\mu} \rho^{\mu}-i \chi\left(\partial_{\mu} \tilde{\rho}^{\mu}-\lambda_{2} \tilde{\chi}\right) \\
& -i \partial_{\mu} K_{\nu}^{\star}\left(\partial^{\mu} K^{\nu}-\partial^{\nu} K^{\mu}\right)+\partial_{\mu} Z^{\star} \partial^{\mu} Z
\end{aligned}
$$

where $K_{\nu}, K_{\nu}^{\star}$ and $Z, Z^{\star}$ are the ghost fields and ghost of ghost fields corresponding to the gaugeon fields. 
Now, the gaugeon fields and respective ghost and ghost of ghost fields changes under the BRST transformation as following:

$$
\begin{aligned}
\delta_{b} Y_{\nu} & =K_{\nu} \delta \lambda, \quad \delta_{b} K_{\nu}=0, \\
\delta_{b} K_{\nu}^{\star} & =i Y_{\nu}^{\star} \delta \lambda, \quad \delta_{b} Y_{\nu}^{\star}=0, \\
\delta_{b} Z^{\star} & =0, \quad \delta_{b} Z=0 .
\end{aligned}
$$

Therefore, the gaugeon Lagrangian density (15) remains intact under the effect of combined BRST transformations (4) and (16). Now we calculate the BRST charge corresponding to lagrangian given in (14) using Noiether theorem, we get

$$
\begin{aligned}
Q_{b} & =\int d^{3} x\left[-2 F^{0 \nu \rho}\left(\partial_{0} \rho_{\nu}-\partial_{\nu} \rho_{0}\right)+\beta_{\nu}\left(\partial^{0} \rho^{\nu}-\partial^{\nu} \rho^{0}\right)-\partial_{\nu} \sigma\left(\partial^{0} \tilde{\rho}^{\nu}-\partial^{\nu} \tilde{\rho}^{0}\right)\right. \\
& \left.+\tilde{\chi} \partial^{0} \sigma-\chi B^{0}-K_{\nu}\left(\partial^{0} Y^{\star \nu}-\partial^{\nu} Y^{\star 0}\right)+Y_{\nu}^{\star}\left(\partial^{0} K^{\nu}-\partial^{\nu} K^{0}\right)\right] .
\end{aligned}
$$

Consequently, the corresponding BRST charge $Q_{b}$ annihilates the physical subspace of $\mathcal{V}_{\text {phys }}$ of total Hilbert space, i.e.

$$
\left.Q_{b} \mid \text { phys }\right\rangle=0 .
$$

This single subsidiary condition of Kugo-Ojima type removes both the unphysical gauge modes as well as unphysical gaugeon modes.

The gaugeon Lagrangian density (15) also admits the following quantum gauge transformations:

$$
\begin{aligned}
& B_{\mu \nu} \longrightarrow \hat{B}_{\mu \nu}=B_{\mu \nu}+\tau\left(\partial_{\mu} Y_{\nu}-\partial_{\nu} Y_{\mu}\right), \quad \rho_{\mu} \longrightarrow \hat{\rho}_{\mu}=\rho_{\mu}+\tau K_{\mu}, \\
& \sigma \longrightarrow \hat{\sigma}=\sigma, \quad \tilde{\rho}_{\mu} \longrightarrow \hat{\tilde{\rho}}_{\mu}=\tilde{\rho}_{\mu}, \quad \beta_{\mu} \longrightarrow \hat{\beta}_{\mu}=\beta_{\mu}, \\
& \tilde{\sigma} \longrightarrow \hat{\tilde{\sigma}}=\tilde{\sigma}, \quad \tilde{\chi} \longrightarrow \hat{\tilde{\chi}}=\tilde{\chi}, \quad \varphi \longrightarrow \hat{\varphi}=\varphi, \quad \chi \longrightarrow \hat{\chi}=\chi, \\
& Y_{\nu} \longrightarrow \hat{Y}_{\nu}=Y_{\nu}, \quad Y_{\nu}^{\star} \longrightarrow \hat{Y}_{\nu}^{\star}=Y_{\nu}^{\star}-\tau \beta_{\nu}, \quad K_{\nu}^{\star} \longrightarrow \hat{K}_{\nu}^{\star}=K_{\nu}^{\star}-\tau \tilde{\rho}_{\mu}, \\
& K_{\nu} \longrightarrow \hat{K}_{\nu}=K_{\nu}, \quad Z \longrightarrow \hat{Z}=Z, \quad Z^{\star} \longrightarrow \hat{Z}^{\star}=Z .
\end{aligned}
$$

Under quantum gauge transformation(19), Lagrangian density (15) remains form invariant, i.e.,

$$
\mathcal{L}\left(\phi^{A}, \alpha\right)=\mathcal{L}\left(\hat{\phi}^{A}, \hat{\alpha}\right),
$$

where

$$
\hat{\alpha}=\alpha+\tau \alpha .
$$

We observe that the quantum gauge transformations (in 18) commute with BRST transformations mentioned in (16). Consequently, it is confirmed that the Hilbert space spanned from physical states annihilated by BRST charge is also invariant under the quantum gauge transformations,

$$
\hat{Q}_{b}=Q_{b} .
$$

Hence physical subspace $\hat{\mathcal{V}}_{\text {phys }}$ is also invariant under quantum gauge transformation.

\section{FFBRST TRANSFORMATION}

In this section, we briefly review the basic mechanism of FFBRST formulation [38]. To do so, let us begin with the BRST transformations (7) and (16) written collectively for all fields $\phi$ of reducible gauge theory,

$$
\delta_{b} \phi=s_{b} \phi \delta \lambda
$$


where $s_{b}$ is Slavnov variation and $\delta \lambda$ is Grassmann parameter of transformation.

Now, we make all the fields $\phi$ a parameter transformation $\kappa$ dependent such that it transform from $\kappa=0$ to $\kappa=1$. Then we make the infinitesimal transformation (23) field-dependent as follows

$$
\frac{d \phi(x, \kappa)}{d \kappa}=s_{b}[\phi(x, \kappa)] \Theta^{\prime}[\phi(x, \kappa)],
$$

where the $\Theta^{\prime}[\phi(x, \kappa)]$ is an infinitesimal field-dependent parameter. After integrating the above transformation from $\kappa=0$ to $\kappa=1$, we get the FFBRST transformation $\left(\delta_{f}\right)$ as follows:

$$
\delta_{f} \phi(x) \equiv \phi(x, \kappa=1)-\phi(x, \kappa=0)=s_{b}[\phi(x)] \Theta[\phi(x)],
$$

where $\Theta[\phi(x)]$ is the finite field-dependent parameter. This is well-known that under resulting FFBRST transformation, the effective action does not change but the functional measure changes drastically with non-trivial Jacobian.

The Jacobian, $J(\kappa)$, of the functional measure $(\mathcal{D} \phi)$ under such transformations after choosing a particular finite field-dependent parameter, $\Theta[\phi(x)]$, can be calculated by writing:

$$
\mathcal{D} \phi^{\prime}=J(\kappa) \mathcal{D} \phi(\kappa) .
$$

The infinitesimal change in Jacobian, $J(\kappa)$, is evaluated by

$$
\frac{1}{J(k)} \frac{d J(k)}{d k}=-\sum_{\phi} \int d^{4} x\left[ \pm\left(s_{b} \phi\right) \frac{\delta \Theta^{\prime}}{\delta \phi}\right],
$$

where + and - sign are used for bosonic and fermionic fields respectively. After integration, this gives [39, 43]

$$
J[\phi]=\exp \left(-\int d^{4} x \sum_{\phi} \pm s_{b} \phi(x) \frac{\delta \Theta^{\prime}[\phi(x)]}{\delta \phi(x)}\right) .
$$

This non-trivial (local) Jacobian gives an extra contribution to generating functional as following:

$$
\int \mathcal{D} \phi^{\prime} e^{i \int d^{4} x \mathcal{L}_{\text {eff }}^{L}\left[\phi^{\prime}\right]}=\int J[\phi] \mathcal{D} \phi e^{i \int d^{4} x \mathcal{L}_{\text {eff }}^{L}[\phi]}=\int \mathcal{D} \phi e^{i \int d^{4} x\left[\mathcal{L}_{\text {eff }}^{L}[\phi]+i \sum_{\phi} \pm s_{b} \phi \frac{\delta \Theta^{\prime}}{\delta \phi}\right]} .
$$

Thus, we see that Jacobian extends the original action by some extra terms.

\section{FFBRST SYMMETRIC GAUGEON FORMALISM}

To study the FFBRST symmetric Gaugeon formalism, we construct the FFBRST transformation, which leaves action (15) invariant, as follows:

$$
\begin{aligned}
\delta_{b} B_{\mu \nu} & =\left(\partial_{\mu} \rho_{\nu}-\partial_{\nu} \rho_{\mu}\right) \Theta[\phi], \quad \delta_{b} \rho_{\mu}=-i \partial_{\mu} \sigma \Theta[\phi], \\
\delta_{b} \sigma & =0, \quad \delta_{b} \tilde{\rho}_{\mu}=i \beta_{\mu} \Theta[\phi], \quad \delta_{b} \beta_{\mu}=0 \\
\delta_{b} \tilde{\sigma} & =\tilde{\chi} \Theta[\phi], \quad \delta_{b} \tilde{\chi}=0, \quad \delta_{b} \varphi=\chi \Theta[\phi], \\
\delta_{b} \chi & =0, \quad \delta_{b} Y_{\nu}=K_{\nu} \Theta[\phi], \quad \delta_{b} K_{\nu}=0, \\
\delta_{b} K_{\nu}^{\star} & =i Y_{\nu}^{\star} \Theta[\phi], \quad \delta_{b} Y_{\nu}^{\star}=0, \quad \delta_{b} Z^{\star}=0, \quad \delta_{b} Z=0,
\end{aligned}
$$

Where $\Theta[\phi]$ is finite field dependent parameter obtainable from the following infinitesimal field-dependent parameter:

$$
\Theta^{\prime}[\phi]=\int d^{3} x\left[-\bar{\rho}_{\mu} \frac{\beta^{\mu}}{\beta_{\rho}^{2}}\left(Y_{\nu}^{*}\right)^{2}-\epsilon \alpha^{2} \bar{\rho}_{\mu} \frac{\beta^{\mu}}{\beta_{\rho}^{2}}\left(\beta_{\nu}\right)^{2}-\epsilon \bar{\rho}_{\mu} \frac{\beta^{\mu}}{\beta_{\rho}^{2}}\left(Y_{\nu}^{*} \beta^{\nu}\right)-\bar{\rho}_{\mu} \frac{\beta^{\mu}}{\beta_{\rho}^{2}}\left(\partial_{\eta} Y_{\nu}^{*}-\partial_{\nu} Y_{\eta}^{*}\right) \partial^{\eta} Y^{\nu}\right] .
$$


We have calculated the Jacobian of functional integral as

$$
J[\phi]=e^{-\int d^{4} x \sum_{\phi} \pm s_{b} \phi(x) \frac{\delta \Theta^{\prime}[\phi(x)]}{\delta \phi(x)}}=e^{i\left[\left(Y_{\nu}^{*}\right)^{2}+\epsilon \alpha^{2}\left(\beta_{\nu}\right)^{2}+2 \epsilon \alpha\left(Y_{\mu}^{*} \beta^{\mu}\right)-\left(\partial_{\mu} Y_{\nu}^{*}-\partial_{\nu} Y_{\mu}^{*}\right) \partial^{\mu} Y^{\nu}\right]} .
$$

This Jacobian changes the action within functional integral as

$$
\int \mathcal{D} \phi^{\prime} e^{i \int d^{4} x \mathcal{L}_{\text {eff }}^{L}\left[\phi^{\prime}\right]}=\int \mathcal{D} \phi e^{i \int d^{4} x\left[\mathcal{L}_{\text {eff }}^{L}[\phi]+i \sum_{\phi} \pm s_{b} \phi \frac{\delta \Theta^{\prime}}{\delta \phi}\right]}=\int \mathcal{D} \phi^{\prime} e^{i \int d^{4} x \mathcal{L}_{B Y}[\phi]}
$$

Since relation

$$
\mathcal{L}_{e f f}^{L}[\phi]+i \sum_{\phi} \pm s_{b} \phi \frac{\delta \Theta^{\prime}}{\delta \phi}=\mathcal{L}_{B Y}[\phi]
$$

can easily be verified by (2), (32) and (15).

Hence in this way, we show that FFBRST formulation is a way to generate the gaugeon modes in a reducible gauge theory naturally.

\section{CONCLUSIONS}

Starting from the most general gauge-fixing Lagrangian including the gaugeon fields, we have presented a general form of the BRST symmetric gaugeon formalism for the 2-form (reducible) gauge theory. This most general gauge-fixing Lagrangian possesses the quantum gauge symmetry under which the Lagrangian remains form invariant. The theory contains two gauge parameters in which one gets shifted by the quantum gauge transformation. We have found that the gaugeon action follows two subsidiary conditions. By introducing Faddeev-Popov ghosts and ghosts of ghosts corresponding to the gaugeon fields, we have constructed a BRST symmetric gaugeon formalism for Abelian 2-form gauge theory. The BRST symmetry enables us to improve the Yokoyama's subsidiary conditions by replacing them to a single Kugo-Ojima type subsidiary condition which is more acceptable. The quantum gauge transformation commutes with the BRST transformation. As a result, the BRST charge is invariant, and thus the physical subspace is also gauge invariant. We have generalized the BRST symmetry of the gaugeon action by making transformation parameter finite and field dependent which still leaves action invariant. But, the functional measure is not invariant under such FFBRST transformations and leads to nontrivial Jacobian. Finally, we have shown that gaugeon fields can be introduced naturally in reducible gauge theory using FFBRST transformation. Although the present paper deals with the Abelian 2-form gauge theory only, these results are more general and will be valid for all reducible gauge theories. In this context, it will be interesting to generalize this result to non-Abelian gauge theory.

[1] K. Yokoyama, Prog. Theor. Phys. 51, 1956 (1974).

[2] K. Yokoyama, Prog. Theor. Phys. 59, 1699 (1978).

[3] K. Yokoyama, Prog. Theor. Phys. 60, 1167 (1978); Phys. Lett. B 79, 79 (1978).

[4] K. Yokoyama and R. Kubo, Prog. Theor. Phys. 52, 290 (1974).

[5] K. Yokoyama, M. Takeda and M. Monda, Prog. Theor. Phys. 60, 927 (1978).

[6] K. Yokoyama, M. Takeda and M. Monda, Prog. Theor. Phys. 64, 1412 (1980).

[7] M. Hayakawa and K Yokoyama, Prog. Theor. Phys. 44, 533 (1970).

[8] K. Izawa, Prog. Theor. Phys. 88, 759 (1992).

[9] M. Koseki, M. Sato and R. Endo, Prog. Theor. Phys. 90, 1111 (1993).

[10] T. Kugo and I. Ojima, Prog. Theor. Phys. Supplement No. 66, 1 (1979).

[11] T. Kugo, I. Ojima, Nucl.Phys. B 144, 234 (1978).

[12] M. Koseki, M. Sato and R. Endo, Bull. of Yamagata Univ., Nat. Sci. 14, 15 (1996).

[13] Y. Nakawaki, Prog. Theor. Phys. 98, 5 (1997). 
[14] R. Endo and M. Koseki, Prog. Theor. Phys. 103, 3, (2000).

[15] H. Miura and R Endo, Prog. Theor. Phys. 117, 4, (2007).

[16] M. Faizal, Commun. Theor. Phys. 57, 637 (2012).

[17] M. Faizal, Mod. Phys. Lett. A 27, 1250147 (2012).

[18] S. Upadhyay, EPL 105, 21001 (2014); Eur. Phys. J. C 74, 2737 (2014); Annals of Physics 344, 290 (2014).

[19] S. Upadhyay and P. K. Panigrahi, Nucl. Phys. B 915, 168 (2017).

[20] M. Kalb and P. Ramond, Phys. Rev. D 9, 22731974.

[21] F. Lund and T. Regge, Phys. Rev. D 14, 1524 (1976); M. Sato and S. Yahikozawa, Nucl. Phys. B436, 100 (1995).

[22] A. Sugamoto, Phys. Rev. D 19, 1820 (1979); R. L. Davis and E. P. S. Shellard, Phys. Lett. B 214,219 (1988).

[23] A. Salam and E. Sezgin, Supergravities in Diverse Dimensions (North-Holland, Amsterdam and World Scientific, Singapore, 1989).

[24] M. B. Green, J. H. Schwarz, and E. Witten, Superstring Theory (Cambridge University Press, New York, 1987); J. Polchinski, String Theory (Cambridge University Press, New York, 1998);

[25] E. Cremmer and J. Scherk, Nucl. Phys. B72, 117 (1974); A. Aurilia and Y. Takahashi, Prog. Theor. Phys. 66, 693 (1981); I. Oda and S. Yahikozawa, ibid. 83, 991 (1990); T. J. Allen, M. J. Bowick and A. Lahiri, Mod. Phys. Lett. A 6, 559 (1991); S. Deguchi, T. Mukai and T. Nakajima, Phys. Rev. D59, 6500359 (1999).

[26] P. G. O. Freund and R. I. Nepomechie, Nucl. Phys. B199, 482 (1982); J. A. de Azcarraga, J. M. Izquierdo, and P. K. Townsend, Phys. Rev. D 45, R3321 (1992); S. Deguchi and T. Nakajima, Int. J. Mod. Phys. A 9 , 1889 (1994).

[27] P. K. Townsend, Phys. Lett. 88B, 97 (1979).

[28] T. Kimura, Prog. Theor. Phys. 64, 357 (1980); H. Hata, T. Kugo, and N. Ohta, Nucl. Phys. B178, 527 (1981); M. Henneaux and C. Teitelboim, Quantization of Gauge Systems (Princeton University Press, Princeton, 1992); J. Gomis, J. Paris, and S. Samuel, Phys. Rep. 259, 1 (1995).

[29] J. Thierry-Mieg and L. Baulieu, Nucl. Phys. B228, 259 (1983); J. Barcelos-Neto and R. Thibes, J. of Math. Phys 39, 5669 (1998).

[30] M. B. Green, J. H. Schwarz and E. Witten, Superstring Theory (Cambridge Univ. Press, 1987).

[31] M. Henneaux and C. Teitelboim, Quantization of gauge systems (Princeton, USA: Univ. Press, 1992).

[32] S. Weinberg, The quantum theory of fields, Vol-II: Modern applications (Cambridge, UK Univ. Press, 1996).

[33] S. Upadhyay, Int. J. Mod. Phys. A 31, 1650148 (2016); Int. J. Mod. Phys. A 31, 1650112 (2016); Int. J. Theor. Phys. 55, 4005 (2016); Eur. Phys. J. C 75, 593 (2015); Phys. Rev. D 92, 065027 (2015); Prog. Theor. Exp. Phys. 093B06, 1 (2015); Int. J. Mod. Phys. A 30, 1550150 (2015); Phys. Lett. B 723, 470 (2013); EPL 103, 61002 (2013); M. Faizal and S. Upadhyay, Phys. Lett. B 736, 288 (2014); S. Upadhyay, M. K. Dwivedi and B. P. Mandal, Int. J. Theor. Phys. 54, 2076 (2015); S. Upadhyay and B. P. Mandal, Eur. Phys. J. C 72, 2059 (2012).

[34] K. Abhinav, A. Shukla, P. K. Panigrahi, Eur. Phys. J. C 76, 639 (2016).

[35] R. Kumar, S. Krishna, A. Shukla, R. P. Malik, Int. J. Mod. Phys. A 29, 1450135 (2014)

[36] S. Upadhyay and B. P. Mandal, Int. J. Mod. Phys. A 28, 1350122 (2013); Eur. Phys. J. C 71, 1759 (2011).

[37] S. D. Joglekar and B. P. Mandal, Phys. Rev. D 51, 1919 (1995).

[38] S. Upadhyay, Phys. Lett. B 740, 341 (2015); Annls. Phys. 356, 299 (2015); Mod. Phys. Lett. A 30,1550072 (2015); Annls. Phys. 340, 110 (2014); arXiv:1308.0982 [hep-th]; EPL 104, 61001 (2013); Phys. Lett. B 727, 293 (2013).

[39] S. Upadhyay and B. Paul, Eur. Phys. J. C 76, 394 (2016); S. Upadhyay, M. Oksanen and R. Bufalo, arXiv:1510.00188 [hep-th].

[40] R. Banerjee and S. Upadhyay, Phys. Lett. B 734, 369 (2014); S. Upadhyay, M. Faizal and P. A. Ganai, Int. J. Mod. Phys. A 30, 1550185 (2015).

[41] R. Banerjee, B. Paul and S. Upadhyay, Phys. Rev. D88, 065019 (2013)

[42] S. Upadhyay and D. Das, Phys. Lett. B 733, 63 (2014).

[43] S. Upadhyay and P. A. Ganai, Prog. Theor. Exp. Phys. 063 B04 (2016) arXiv:1605.04290.

[44] S. Upadhyay and B. P. Mandal, Eur. Phys. J. C 75, 327 (2015); Int. J. Theor. Phys. 55, 1 (2016); Phys. Lett. B 744, 231 (2015); Prog. Theor. Exp. Phys. 053B04 (2014); Eur. Phys. J. C 72, 2065 (2012); Annls. Phys. 327, 2885 (2012); AIP Conf. Proc. 1444, 213 (2012); Eur. Phys. Lett. 93, 31001 (2011); Mod. Phys. Lett. A 25, 3347 (2010); B. P. Mandal, S. K. Rai and S. Upadhyay, EPL 92, 21001 (2010); S. Upadhyay, M. K. Dwivedi and B. P. Mandal, Int. J. Mod. Phys. A 30, 1550178 (2015); Int. J. Mod. Phys. A 28, 1350033 (2013).

[45] M. Faizal, B. P. Mandal and S. Upadhyay, Phys. Lett. B 721, 159 (2013); M. Faizal, S. Upadhyay and B. P. Mandal, Phys. Lett. B 738, 201 (2014).

[46] M. Faizal, S. Upadhyay and B. P. Mandal, Eur. Phys. J. C 76, 189 (2016).

[47] S. Upadhyay, A. Reshetnyak and B. P. Mandal, Eur. Phys. J. C 76, 391 (2016). 
[48] P. Y. Moshin and A. A. Reshetnyak, Int. J. Mod. Phys. A 30, 1550021 (2015).

[49] P. Y. Moshin and A. A. Reshetnyak, Phys. Lett. B 739, 110 (2014).

[50] P. Y. Moshin and A. A. Reshetnyak, arXiv: 1506.04660[hep-th]. 